\title{
ESTRUCTURA Y DIVERSIDAD DEL MATORRAL SUBMONTANO CONTIGUO AL ÁREA METROPOLITANA DE MONTERREY, NUEVO LEÓN, MÉXICO
}

\begin{abstract}
Eduardo Alanís-Rodríguez ${ }^{1,6}$, Javier Jiménez-Pérez ${ }^{1}$, Arturo Mora-Olivo², José Guadalupe Martínez-Ávalos ${ }^{2}$, José Manuel Mata-Balderas ${ }^{3}$, Alejandro Collantes Chávez-Costa ${ }^{4}$ y Ernesto A. Rubio-Camacho 5
\end{abstract}

${ }^{1}$ Universidad Autónoma de Nuevo León, Facultad de Ciencias Forestales, Carretera Linares-Cd. Victoria km 145, Apdo. postal 41, 67700 Linares, Nuevo León, México.

${ }^{2}$ Universidad Autónoma de Tamaulipas, Instituto de Ecología Aplicada, División del Golfo 356, 87019 Ciudad Victoria, Tamaulipas, México.

${ }^{3}$ Gestión Estratégica y Manejo Ambiental S.C., Carretera San Miguel-Huinalá 935, Tercer Piso, Local 34, Plaza Comercial Acanto, 66647 Apodaca, Nuevo León, México.

${ }^{4}$ Universidad de Quintana Roo, División de Desarrollo Sustentable, Avenida Andrés Quintana Roo con calle 110 Sur s/n, 77600 Cozumel, Quintana Roo, México. ${ }^{5}$ Campo Experimental Centro-Altos de Jalisco, Centro de Investigación Regional Pacífico Centro, INIFAP, km 8 Carretera libre Tepatitlán-Lagos de Moreno. Apartado postal 56, 47600. Jalisco, México.

${ }^{6}$ Autor para la correspondencia: eduardo.alanisrd@uanl.edu.mx

\section{RESUMEN}

En este estudio se evaluaron la estructura y la diversidad del matorral submontano limítrofe con la porción occidental del área metropolitana de Monterrey, México. Se determinó la composición y la diversidad de la vegetación mediante el establecimiento de 41 parcelas de $100 \mathrm{~m}^{2}$ cada una. Se censaron todos los árboles y arbustos con un diámetro basal $\left(d_{0.10}\right) \geq 0.5 \mathrm{~cm}$, incluyendo a las plantas suculentas; además, se midió el diámetro de copa $\left(d_{\text {сора }}\right)$, para cada especie. Se calcularon los índices de Margalef $\left(\mathrm{D}_{\mathrm{Mg}}\right)$ y de Shannon $\left(\mathrm{H}^{\prime}\right)$ para determinar la riqueza y la diversidad de especies, respectivamente. Se registraron 45 especies, 39 géneros y 18 familias. La familia Fabaceae fue la que presentó más taxones (12), seguida de Cactaceae (5), Euphorbiacae, Rhamnaceae, Rutaceae y Verbenaceae (3), las cuales constituyen en conjunto $64.44 \%$ de la flora registrada para 
la zona de estudio. En cuanto a la dominancia $\left(\mathrm{D}_{\mathrm{r}}\right)$; las Fabaceae, Rutaceae, Asteraceae y Verbenaceae sumaron $64.08 \%$ de los valores del Índice de Valor de Importancia (IVI) en la comunidad. Este matorral presenta una densidad de 3629 ind./ha ${ }^{-1}$ y una cobertura de copas de $6629 \mathrm{~m}^{2} / \mathrm{ha}^{-1}$. Acacia amentacea y Zanthoxylum fagara fueron las más importantes al acumular entre ellas $25.87 \%$ de los valores del IVI en la localidad. La curva de rango/abundancia de especies se ajustó a una función log-normal, característica de la vegetación madura. La comunidad estudiada presenta una alta diversidad y riqueza de especies en comparación con otras asociaciones vegetales de climas áridos y semiáridos del noreste de México. Los atributos de estructura y diversidad documentados en este estudio indican que el matorral submontano contiguo a la ciudad de Monterrey está bien conservado y en una fase de sucesión madura, por lo que es urgente proponer estrategias para su conservación.

Palabras clave: comunidad vegetal, riqueza florística, vegetación xerófila, vegetación periurbana.

\section{ABSTRACT}

In this study the structure and diversity of the piedmont scrub that abuts the western portion of the metropolitan area of Monterrey, Mexico, were examined. Vegetation composition and diversity were assessed by sampling vegetation in 41 plots of $100 \mathrm{~m}^{2}$ each. All trees and shrubs with a basal diameter $\left(d_{0.10}\right) \geq 0.5 \mathrm{~cm}$ were counted, including succulents. In addition, the crown diameter $\left(d_{\text {crown }}\right)$ for each species was recorded. Species richness was assessed by means of the Margalef index $\left(\mathrm{D}_{\mathrm{Mg}}\right)$, while Shannon's index $\left(\mathrm{H}^{\prime}\right)$ was used to assess species diversity. A total of 45 species, 39 genera and 18 families were recorded. Fabaceae was the most important family with the highest species richness (12), followed by Cactaceae (5), Euphorbiacae, Rhamnaceae, Rutaceae and Verbenaceae (3); these families accounted for $64.44 \%$ of the entire flora recorded in the area. As for dominance $\left(\mathrm{D}_{\mathrm{r}}\right)$, Fabaceae, Rutaceae, Asteraceae and Verbenaceae accounted for $64.08 \%$ of the Importance Value Index (IVI) in the community. The scrub presented a density of $3629 \mathrm{ind} . / \mathrm{ha}^{-1}$ and a total crown cover of $6629 \mathrm{~m}^{2} / \mathrm{ha}^{-1}$. Acacia amentacea and Zanthoxylum fagara were the most important species, since they accounted for $25.87 \%$ of the IVI values in the locality. The rank/abundance curve for species fitted best to a log-normal function, which is characteristic of mature communities. The present study shows that the studied community is characterized by a high diversity and species richness in comparison to other arid and semi-arid climate vegetation in Northeast Mexico. Structural and diversity 
attributes documented in this study indicated that the piedmont scrub neighboring Monterrey is a well-preserved community in a mature successional stage; hence, it is urgent to develop strategies aimed at its conservation.

Key words: arid vegetation, floristic richness, peri-urban vegetation, vegetation community.

\section{INTRODUCCIÓN}

El matorral xerófilo es una de las comunidades vegetales más importantes de México, debido a que ocupa alrededor de $40 \%$ de la superficie total del país, además de ser el tipo de vegetación con el mayor número de plantas endémicas y con una riqueza florística calculada en alrededor de 6000 especies (Rzedowski, 1992). Una de las variantes importantes del matorral xerófilo es el matorral submontano, también descrito como "piedmont scrub” por Muller (1939), el cual es una asociación vegetal localizada principalmente en las partes medias y en las faldas de la Sierra Madre Oriental, en los estados de Coahuila, Nuevo León, Tamaulipas, Hidalgo, San Luis Potosí, Querétaro, Puebla y Oaxaca (Briones, 1991; Canizales-Velázquez et al., 2009; Estrada-Castillón et al., 2012; Fernández y Colmenero, 1997; González-Costilla et al., 2007; Gómez et al., 2011; Rzedowski, 1978; Salinas-Rodríguez et al., 2013).

Particularmente en Nuevo León, el matorral submontano ocupa $11 \%$ de la superficie del estado (Palacio-Prieto et al., 2000), donde se desarrolla en altitudes que van de 450 a 800 m s.n.m. (Muller, 1939; Rojas-Mendoza, 1965). Tal tipo de vegetación ha sido estudiado en esta entidad por diversos autores con el propósito de conocer su estructura y composición florística (Canizales-Velázquez et al., 2009; Estrada-Castillón et al., 2012). Sin embargo, es importante conocer con mayor detalle su diversidad y estructura en las zonas cercanas a las franjas urbanas, debido a que los efectos humanos asociados a las grandes ciudades pueden poner en riesgo su permanencia. Diversos autores han puesto de manifiesto la importancia del conocimiento de la heterogeneidad de los paisajes, así como de su fragmentación en diferentes porciones de ecosistemas y de las relaciones que se establecen entre las mismas, como posibles corredores de especies, para poder orientar los esfuerzos dirigidos a la conservación de la biodiversidad (Forman y Godron, 1986; Forman, 1995; Farina, 1998; Naveh y Lieberman, 1984; Spellerberg y Sawyer, 1999; Yablokov y Ostroumov, 1989). 
Las ciudades con más habitantes en el estado de Nuevo León (Monterrey y su área metropolitana, Montemorelos, Santiago, Linares, Allende) se localizan o están rodeadas por el matorral submontano. Estas zonas urbanas han crecido considerablemente en los últimos años (Estrada-Castillón et al., 2012). Tal es el caso del área metropolitana de Monterrey, donde el uso de suelo de las partes montañosas ha cambiado paulatinamente para el establecimiento de nuevos asentamientos. Esta situación se presenta a pesar de que algunas de estas zonas están sujetas a protección ecológica por el gobierno federal o por los gobiernos estatales, como sucede con la Sierra Las Mitras (Anónimo, 2002).

Debido al escaso conocimiento de las comunidades vegetales contiguas al área metropolitana de Monterrey y al intenso cambio de uso de suelo, es necesario generar información cuantitativa de la estructura y la diversidad del matorral submontano. Por lo tanto, el objetivo de la presente investigación fue evaluar la estructura y la diversidad florística del matorral submontano contiguo a la porción occidental del área metropolitana de Monterrey.

\section{MATERIAL Y MÉTODOS}

Área de estudio

El estudio se desarrolló en una comunidad vegetal de matorral submontano con elementos de matorral espinoso tamaulipeco del noreste de México, específicamente en el municipio de García, Nuevo León (Fig. 1). Las coordenadas de ubicación son $25^{\circ} 42^{\prime} 55^{\prime \prime} \mathrm{N}$ y $100^{\circ} 26^{\prime} 05^{\prime \prime} \mathrm{W}$, con una amplitud altitudinal de 640 a 820 m s.n.m. El clima es seco ( $\left.\mathrm{BS}_{0} \mathrm{hw}\right)$, según la clasificación de Köppen modificada por García (1988). La temperatura media anual es de 20 a $22^{\circ} \mathrm{C}$ y los meses más calientes son julio y agosto, mientras que las más bajas se registran en diciembre y enero, con un promedio de 13 a $14{ }^{\circ} \mathrm{C}$. El suelo del área donde se realizó el estudio está constituido por rendzinas.

\section{Trabajo de campo}

En el mes de septiembre de 2012 se evaluó la comunidad vegetal del área de estudio, tomando en consideración los componentes arbóreo y arbustivo, así como a las plantas suculentas. Para determinar el número de parcelas necesarias para obtener información representativa, en septiembre del año 2012 se realizó un pre-muestreo de 20. Con los datos recabados se utilizó el siguiente modelo matemático (Mostacedo y Fredericksen, 2000), tomando en consideración la variable cobertura. 


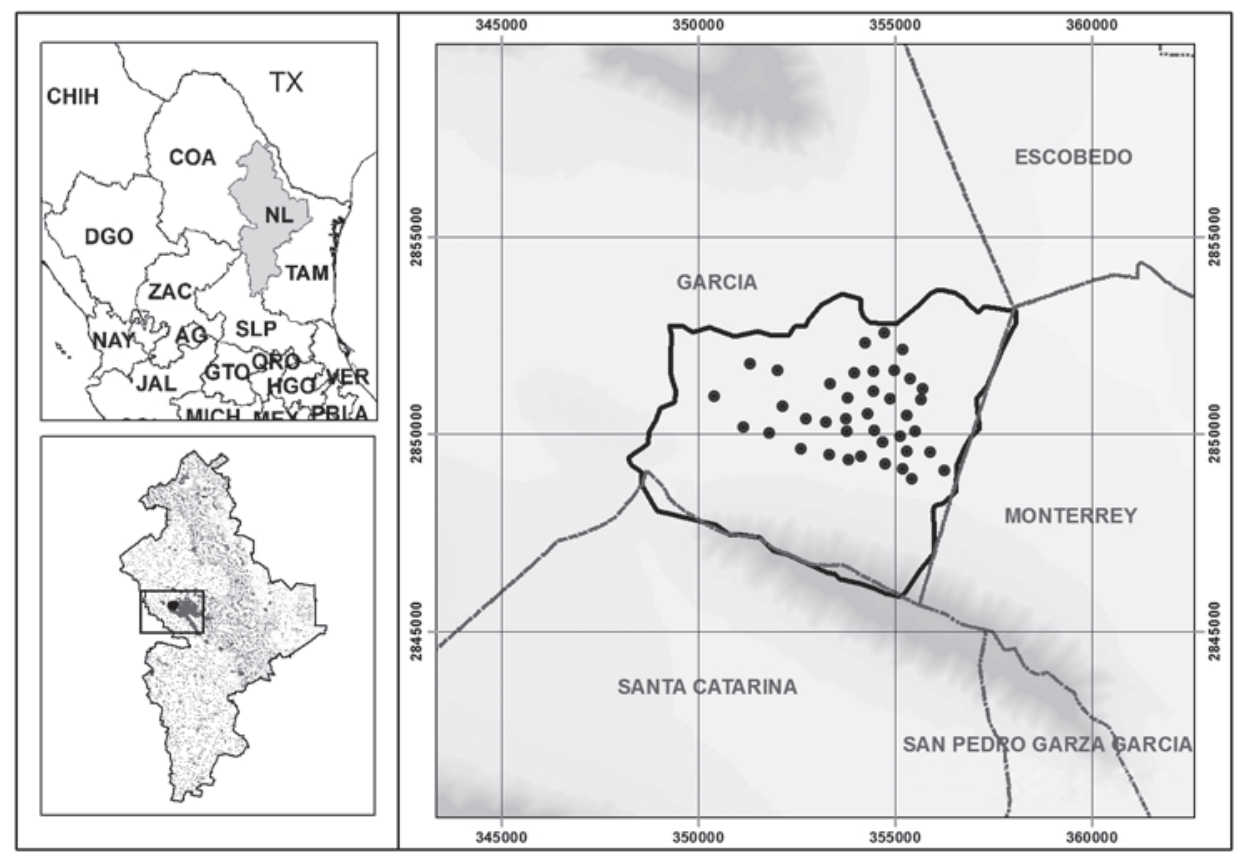

Fig. 1. Ubicación del área de estudio. La imagen superior izquierda muestra el noreste de México y sureste de Estados Unidos, la inferior izquierda el estado de Nuevo León, donde se aprecia la localización de la ciudad de Monterrey y su área metropolitana, y la imagen de la derecha la ubicación espacial de las 41 parcelas.

$$
\mathrm{n}=\frac{\mathrm{t}^{2} * C V^{2}}{\mathrm{E}^{2}+\frac{\mathrm{t}^{2} * C V^{2}}{\mathrm{~N}}}
$$

$n$ = número adecuado de parcelas de muestreo, $E$ = error (20\%), $t=$ valor extraído de las tablas de " $t$ " de Student $(P<0.05), N=$ total de unidades muestrales en toda la población, $C V=$ coeficiente de variación.

Las parcelas de muestreo fueron de $10 \times 10 \mathrm{~m}\left(100 \mathrm{~m}^{2}\right)$. En cada una de ellas se realizó un censo de todos los arbustos y árboles $\geq 0.5 \mathrm{~cm}$ de diámetro basal $\left(d_{0.10}\right)$ así como de plantas suculentas. A todos los individuos se les midió con una cinta métrica el diámetro de copa $\left(d_{\text {сора }}\right)$ en sentido norte-sur y este-oeste. De acuerdo con el resultado del modelo, se establecieron 41 parcelas, lo cual constituye una superficie total evaluada de $4100 \mathrm{~m}^{2}$. 
Análisis de la información

Para determinar la diversidad $\alpha$ se utilizaron dos índices, el de Margalef $\left(D_{\mathrm{Mg}}\right)$ que está basado en la cuantificación del número de especies presentes (riqueza específica), y el de Shannon $\left(H^{\prime}\right)$, el cual refiere a la estructura numérica de la comunidad, es decir, en la distribución proporcional de la abundancia de cada especie (Moreno, 2001). Las fórmulas se describen a continuación:

$$
\begin{gathered}
D_{M g}=\frac{(S-1)}{\ln (N)} \\
H^{\prime}=-\sum_{i=1}^{S} p_{i} \times \ln \left(p_{i}\right) \\
P i=n_{i} / N
\end{gathered}
$$

donde $S$ = número de especies presentes, $N$ = número total de individuos, $n_{\mathrm{i}}=$ número de individuos de la especie $i$.

Para cada especie se determinó su abundancia de acuerdo con el número de individuos, su dominancia en función de la cobertura de copa y su frecuencia con base en su presencia en las parcelas de muestreo. Estos resultados fueron utilizados para obtener un valor ponderado a nivel de taxon denominado Índice de Valor de Importancia (IVI), el cual adquiere valores porcentuales en una escala del 0 al 100 (Mueller-Dombois y Ellenberg, 1974). Para la estimación de la abundancia relativa $\left(A R_{\mathrm{i}}\right)$ de cada especie se empleó la siguiente ecuación:

$$
A R_{i}=\left|/ \sum_{i=1 . \ldots n} A_{i}\right| \times 100
$$

donde $A_{\mathrm{i}}=$ abundancia absoluta. La dominancia relativa $\left(D R_{\mathrm{i}}\right)$ se evaluó mediante la fórmula:

$$
D R_{i}=\left|/ \sum_{i=1 . . . n} D_{i}\right| \times 100
$$

donde $D_{\mathrm{i}}=$ dominancia absoluta. La frecuencia relativa $\left(F R_{\mathrm{i}}\right)$ se obtuvo con la siguiente ecuación: 


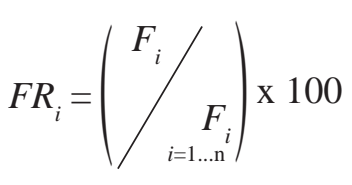

donde $F_{\mathrm{i}}=$ frecuencia absoluta $=P_{\mathrm{i}} / N S, P_{\mathrm{i}}=$ número de sitios en los que está presente la especie $i$, y $N S$ = número total de sitios de muestreo. El IVI se define como (Whittaker, 1972; Moreno, 2001):

$$
I V I=\frac{\sum_{n}^{i=1}\left(A R_{i}, D R_{i}, F R_{i}\right)}{3}
$$

donde $A R_{\mathrm{i}}=$ abundancia relativa de la especie $i$ respecto a la abundancia total, $D R_{\mathrm{i}}=$ dominancia relativa de la especie $i$ respecto a la dominancia total, y $F R_{\mathrm{i}}=$ frecuencia relativa de la especie $i$ respecto a la frecuencia total.

El Índice de Valor de Importancia Familiar (IVIF) adquiere valores de 0 a 100\% y se calculó de la siguiente manera (Whittaker, 1972; Moreno, 2001):

$$
\mathrm{IVIF}=\frac{\sum_{\mathrm{n}}^{\mathrm{i}=1}\left(\mathrm{ARF}_{\mathrm{i}}, \mathrm{DRF}_{\mathrm{i}}, \mathrm{FRF}_{\mathrm{i}}\right)}{3}
$$

donde $A R F_{\mathrm{i}}=$ abundancia relativa de la familia $i$ respecto a la abundancia total, $D R F_{\mathrm{i}=}$ dominancia relativa de la familia $i$ respecto a la dominancia total, y $F R F_{\mathrm{i}}=$ frecuencia relativa de la familia $i$ respecto a la frecuencia total.

Para describir la estructura de la comunidad en términos de la abundancia de cada especie se utilizaron curvas de rango/abundancia (Magurran, 2004). Éstas muestran la relación entre el valor de abundancia absoluta de las especies en función de un arreglo secuencial que van de la más común a la más rara (Martella et al., 2012). Se probaron los modelos de la serie logarítmica $\left(\mathrm{S}_{\mathrm{log}}\right)$, Poissonlognormal y lognormal-truncado. Los parámetros se estimaron con el método de máxima verosimilitud y para la elección del mejor prototipo se utilizaron tres criterios. El primero fue el de información de Akaike (AIC), de acuerdo con el cual se examinan los modelos seleccionados tomando en cuenta su ajuste y su complejidad; cuando se hace la comparación utilizando este método la selección del mejor modelo está en función del menor valor en AIC. Además, se utilizó el criterio de que cuando $\triangle \mathrm{AIC}$ es menor que 2 , esto quiere decir que los modelos en cuestión explican de manera similar la tendencia de los datos, mientras que valores mayores a 2 evidencian una diferencia entre los modelos. El segundo criterio 
fue el logaritmo de la función de verosimilitud (log-likelihood), que da apoyo al del AIC; en este caso se toma el de menor valor para la comparación de modelos. El tercero fue el coeficiente de correlación de Pearson, que se utiliza como un indicador de cuán cerca están los valores observados de los predichos en términos relativos (valores cercanos a 1 indican que hay una correlación fuerte). Este último se utiliza en lugar de la $R^{2}$ (Potts y Elith, 2006), el cual se recomienda pero para los modelos de la serie geométrica y de la vara rota, por presentar ambos una relación lineal en la gráfica de rango/abundancia (Matthews y Whittaker, 2014). Los tres criterios se tomaron en cuenta para la elección del mejor modelo y para dar más apoyo al análisis estadístico e interpretación de los resultados. Éstos se aplicaron a los tres modelos y para la comparación de los mismos se tomó el siguiente orden: (1) el criterio AIC, (2) el logaritmo de la función de verosimilitud, y (3) la correlación. El análisis se llevó a cabo con el programa R v3.1.2 (R Development Core Team, 2011), utilizando las rutinas de Prado y Miranda (2014) y con apoyo de la plataforma RStudio.

\section{RESULTADOS}

\section{Riqueza}

En las 41 parcelas de muestreo se registraron 45 especies, 39 géneros y 18 familias (Apéndice). Las familias con más especies fueron Fabaceae con 12 y Cactaceae, con cinco, Euphorbiacae, Rhamnaceae, Rutaceae y Verbenaceae con tres cada una. Estas seis familias incluyeron 25 géneros y 29 especies, lo que constituye 64.4\% de la flora registrada en el área de estudio.

\section{Estructura}

De acuerdo con los valores del IVIF obtenidos, las familias Fabaceae, Rutaceae, Asteraceae y Verbenaceae fueron las más importantes, sumando 64.1\% del total de la comunidad. Las que presentaron menos de 1\% de IVIF fueron Sapindaceae, Zygophyllaceae, Solanaceae y Simaroubaceae (Cuadro 1).

La comunidad vegetal estudiada presentó una densidad de 3629 ind./ha-1 y una cobertura de copas de $6629 \mathrm{~m}^{2} / \mathrm{ha}^{-1}$. A nivel de especie, Acacia amentacea y Zanthoxylum fagara fueron las más sobresalientes según los valores de IVI, al registrar los más altos, sumando $25.87 \%$ del total de la comunidad. $55.6 \%$ de las especies (25 de 45) presentan valores de IVI inferiores a 1.0\% (Cuadro 2). 
Cuadro 1. Parámetros estructurales estimados para las familias registradas en el área de estudio. IVIF = Índice de Valor de Importancia Familiar.

\begin{tabular}{|c|c|c|c|c|c|c|}
\hline \multirow[t]{2}{*}{ Familia } & \multicolumn{2}{|c|}{ Abundancia } & \multicolumn{2}{|c|}{ Dominancia } & \multirow{2}{*}{$\begin{array}{c}\text { Frecuencia } \\
\text { Relativa } \\
(\%)\end{array}$} & \multirow[t]{2}{*}{ IVIF } \\
\hline & $\begin{array}{l}\text { Absoluta } \\
\text { (ind./ha }{ }^{-1} \text { ) }\end{array}$ & $\begin{array}{c}\text { Relativa } \\
(\%)\end{array}$ & $\begin{array}{l}\text { Absoluta } \\
\left(\mathrm{m}^{2} / \mathrm{ha}^{-1}\right)\end{array}$ & $\begin{array}{c}\text { Relativa } \\
(\%)\end{array}$ & & \\
\hline Fabaceae & 1115 & 30.71 & 2518.04 & 37.99 & 40.52 & 36.41 \\
\hline Rutaceae & 256 & 7.06 & 949.35 & 14.32 & 15.28 & 12.22 \\
\hline Asteraceae & 300 & 8.27 & 488.36 & 7.37 & 7.86 & 7.83 \\
\hline Verbenaceae & 346 & 9.54 & 427.22 & 6.45 & 6.87 & 7.62 \\
\hline Boraginaceae & 237 & 6.52 & 368.14 & 5.55 & 5.92 & 6.00 \\
\hline Euphorbiaceae & 329 & 9.07 & 265.02 & 4.00 & 4.26 & 5.78 \\
\hline Scrophulariaceae & 356 & 9.81 & 423.83 & 6.39 & 0.17 & 5.46 \\
\hline Cactaceae & 80 & 2.22 & 359.29 & 5.42 & 5.78 & 4.47 \\
\hline Agavaceae & 159 & 4.37 & 246.31 & 3.72 & 3.96 & 4.02 \\
\hline Rhamnaceae & 151 & 4.17 & 115.53 & 1.74 & 1.86 & 2.59 \\
\hline Fouquieriaceae & 34 & 0.94 & 165.11 & 2.49 & 2.66 & 2.03 \\
\hline Ulmaceae & 78 & 2.15 & 121.23 & 1.83 & 1.95 & 1.98 \\
\hline Ebenaceae & 73 & 2.02 & 83.17 & 1.25 & 1.34 & 1.54 \\
\hline Oleaceae & 71 & 1.95 & 49.24 & 0.74 & 0.79 & 1.16 \\
\hline Sapindaceae & 22 & 0.60 & 29.98 & 0.45 & 0.48 & 0.51 \\
\hline Zygophyllaceae & 15 & 0.40 & 17.54 & 0.26 & 0.28 & 0.32 \\
\hline Simaroubaceae & 5 & 0.13 & 0.17 & 0.00 & 0.00 & 0.05 \\
\hline Solanaceae & 2 & 0.07 & 0.90 & 0.01 & 0.01 & 0.03 \\
\hline Total & 3629 & 100.00 & 6628.00 & 100.00 & 100.00 & 100.00 \\
\hline
\end{tabular}

Curva de rango/abundancia de las especies

En la Figura 2 se muestra la curva de rango/abundancia para todas las especies registradas en el muestreo de la vegetación. Ésta se ajusta gráficamente a la distribución Poisson-Lognormal y a la Lognormal-truncada, pero no así a la $\mathrm{S}_{\log }$; tales ajustes se confirman con los valores de AIC, que para la Poisson-Lognormal fue de 473.3, $\triangle \mathrm{AIC}=0,(\log -1$ likelihood $=-234.6 ; \mathrm{r}=0.988)$, la Lognormal-Truncada de 473.5, $\triangle \mathrm{AIC}=0.2,($ Log-likelihood $=-234.7 ; \mathrm{r}=0.986)$ y la Serie Logarítmica de 482.6 $\triangle \mathrm{AIC}=9.3$, (Log-likelihood $=-240.3 ; \mathrm{r}=0.986)$. De esta manera se puede describir la abundancia de especies en el área utilizando el modelo de Poisson- 
Cuadro 2. Parámetros estructurales estimados para las especies registradas en el área de estudio. IVI = Índice de Valor de Importancia.

\begin{tabular}{|c|c|c|c|c|c|c|c|}
\hline \multirow[t]{2}{*}{ Especie } & \multicolumn{2}{|c|}{ Abundancia } & \multicolumn{2}{|c|}{ Dominancia } & \multicolumn{2}{|c|}{ Frecuencia } & \multirow[t]{2}{*}{ IVI } \\
\hline & $\begin{array}{l}\text { Absoluta } \\
\text { (ind/ha }^{-1} \text { ) }\end{array}$ & $\begin{array}{c}\text { Relativa } \\
(\%)\end{array}$ & $\begin{array}{l}\text { Absoluta } \\
\left(\mathrm{m}^{2} / \mathrm{ha}^{-1}\right)\end{array}$ & $\begin{array}{c}\text { Relativa } \\
(\%)\end{array}$ & Absoluta & $\begin{array}{c}\text { Relativa } \\
\text { (\%) }\end{array}$ & \\
\hline Acacia amentacea & 607 & 16.73 & 855.54 & 12.91 & 31.48 & 12.91 & 14.18 \\
\hline Zanthoxylum fagara & 246 & 6.79 & 937.45 & 14.14 & 34.49 & 14.14 & 11.69 \\
\hline $\begin{array}{l}\text { Gymnosperma } \\
\text { glutinosum }\end{array}$ & 300 & 8.27 & 488.36 & 7.37 & 17.97 & 7.37 & 7.67 \\
\hline Leucophyllum frutescens & 356 & 9.81 & 423.83 & 6.39 & 15.60 & 6.39 & 7.53 \\
\hline Acacia berlandieri & 217 & 5.98 & 435.58 & 6.57 & 16.03 & 6.57 & 6.37 \\
\hline Cordia boissieri & 222 & 6.12 & 360.18 & 5.43 & 13.25 & 5.43 & 5.66 \\
\hline Caesalpinia mexicana & 20 & 0.54 & 497.40 & 7.50 & 18.30 & 7.50 & 5.18 \\
\hline Eysenhardtia texana & 90 & 2.49 & 398.47 & 6.01 & 14.66 & 6.01 & 4.84 \\
\hline Lantana macropoda & 217 & 5.98 & 270.18 & 4.08 & 9.94 & 4.08 & 4.71 \\
\hline Bernardia myricifolia & 276 & 7.59 & 203.93 & 3.08 & 7.50 & 3.08 & 4.58 \\
\hline Opuntia engelmannii & 49 & 1.34 & 333.18 & 5.03 & 12.26 & 5.03 & 3.80 \\
\hline Lippia graveolens & 129 & 3.56 & 157.04 & 2.37 & 5.78 & 2.37 & 2.77 \\
\hline Agave lecheguilla & 90 & 2.49 & 174.83 & 2.64 & 6.43 & 2.64 & 2.59 \\
\hline Fouquieria splendens & 34 & 0.94 & 165.11 & 2.49 & 6.08 & 2.49 & 1.97 \\
\hline Celtis pallida & 54 & 1.48 & 105.76 & 1.60 & 3.89 & 1.60 & 1.56 \\
\hline Karwinskia humboldtiana & 85 & 2.35 & 76.54 & 1.15 & 2.82 & 1.15 & 1.55 \\
\hline Yucca filifera & 68 & 1.88 & 71.48 & 1.08 & 2.63 & 1.08 & 1.35 \\
\hline Sophora secundiflora & 44 & 1.21 & 70.65 & 1.07 & 2.60 & 1.07 & 1.11 \\
\hline Forestiera angustifolia & 66 & 1.81 & 44.14 & 0.67 & 1.62 & 0.67 & 1.05 \\
\hline Havardia pallens & 51 & 1.41 & 53.77 & 0.81 & 1.98 & 0.81 & 1.01 \\
\hline Ebenopsis ebano & 10 & 0.27 & 87.43 & 1.32 & 3.22 & 1.32 & 0.97 \\
\hline Condalia hookeri & 61 & 1.68 & 31.75 & 0.48 & 1.17 & 0.48 & 0.88 \\
\hline Diospyros palmeri & 2 & 0.07 & 82.09 & 1.24 & 3.02 & 1.24 & 0.85 \\
\hline Acacia greggii & 29 & 0.81 & 49.25 & 0.74 & 1.81 & 0.74 & 0.76 \\
\hline Jatropha dioica & 37 & 1.01 & 40.32 & 0.61 & 1.48 & 0.61 & 0.74 \\
\hline Diospyros texana & 71 & 1.95 & 1.08 & 0.02 & 0.04 & 0.02 & 0.66 \\
\hline Citharexylum berlandieri & 22 & 0.60 & 29.98 & 0.45 & 1.10 & 0.45 & 0.50 \\
\hline Celtis laevigata & 24 & 0.67 & 15.47 & 0.23 & 0.57 & 0.23 & 0.38 \\
\hline Croton ciliatoglandulifer & 17 & 0.47 & 20.77 & 0.31 & 0.76 & 0.31 & 0.37 \\
\hline Senna bauhinioides & 22 & 0.60 & 11.83 & 0.18 & 0.44 & 0.18 & 0.32 \\
\hline
\end{tabular}


Cuadro 2. Continuación

\begin{tabular}{|c|c|c|c|c|c|c|c|}
\hline \multirow[t]{2}{*}{ Especie } & \multicolumn{2}{|c|}{ Abundancia } & \multicolumn{2}{|c|}{ Dominancia } & \multicolumn{2}{|c|}{ Frecuencia } & \multirow[t]{2}{*}{ IVI } \\
\hline & $\begin{array}{l}\text { Absoluta } \\
\left(\text { ind/ha }^{-1} \text { ) }\right.\end{array}$ & $\begin{array}{l}\text { Relativa } \\
(\%)\end{array}$ & $\begin{array}{l}\text { Absoluta } \\
\left(\mathrm{m}^{2} / \mathrm{ha}^{-1}\right)\end{array}$ & $\begin{array}{l}\text { Relativa } \\
(\%)\end{array}$ & Absoluta & $\begin{array}{c}\text { Relativa } \\
(\%)\end{array}$ & \\
\hline Guaiacum angustifolium & 15 & 0.40 & 17.54 & 0.26 & 0.65 & 0.26 & 0.31 \\
\hline Prosopis laevigata & 2 & 0.07 & 28.38 & 0.43 & 1.04 & 0.43 & 0.31 \\
\hline $\begin{array}{l}\text { Ferocactus } \\
\text { haematacanthus }\end{array}$ & 12 & 0.34 & 15.94 & 0.24 & 0.59 & 0.24 & 0.27 \\
\hline Acacia farnesiana & 7 & 0.20 & 19.42 & 0.29 & 0.71 & 0.29 & 0.26 \\
\hline Mimosa malacophylla & 15 & 0.40 & 10.30 & 0.16 & 0.38 & 0.16 & 0.24 \\
\hline Ehretia anacua & 15 & 0.40 & 7.96 & 0.12 & 0.29 & 0.12 & 0.21 \\
\hline $\begin{array}{l}\text { Echinocereus } \\
\text { enneacanthus }\end{array}$ & 12 & 0.34 & 7.34 & 0.11 & 0.27 & 0.11 & 0.19 \\
\hline Helietta parvifolia & 7 & 0.20 & 9.37 & 0.14 & 0.34 & 0.14 & 0.16 \\
\hline Ziziphus obtusifolia & 5 & 0.13 & 7.25 & 0.11 & 0.27 & 0.11 & 0.12 \\
\hline Fraxinus greggii & 5 & 0.13 & 5.10 & 0.08 & 0.19 & 0.08 & 0.10 \\
\hline Mammillaria prolifera & 5 & 0.13 & 1.61 & 0.02 & 0.06 & 0.02 & 0.06 \\
\hline Casimiroa greggii & 2 & 0.07 & 2.53 & 0.04 & 0.09 & 0.04 & 0.05 \\
\hline $\begin{array}{l}\text { Castela erecta subsp. } \\
\text { texana }\end{array}$ & 5 & 0.13 & 0.17 & 0.00 & 0.01 & 0.00 & 0.05 \\
\hline $\begin{array}{l}\text { Echinocereus } \\
\text { pentalophus }\end{array}$ & 2 & 0.07 & 1.23 & 0.02 & 0.05 & 0.02 & 0.03 \\
\hline Nicotiana glauca & 2 & 0.07 & 0.90 & 0.01 & 0.03 & 0.01 & 0.03 \\
\hline Total & 3629 & 100 & 6628 & 100.00 & 244.00 & 100.00 & 100.00 \\
\hline
\end{tabular}

Lognormal o la distribución Lognormal-truncada, ya que la distribución de especies se ajusta a los dos modelos tanto gráfica como analíticamente. Sin embargo, la Poisson-lognormal mostró, aunque por un margen escaso, los mejores ajustes. En este caso utilizamos la distribución de Poisson-lognormal, ya que esta distribución está documentada para comunidades vegetales maduras (Long et al., 2012; Martella et al., 2012; Matthews y Whittaker, 2014).

\section{Diversidad}

La comunidad vegetal del matorral submontano estudiado presentó un índice de Margalef de 6.02. En lo que respecta a la diversidad de especies, el valor del índice de Shannon fue de 3.02. 


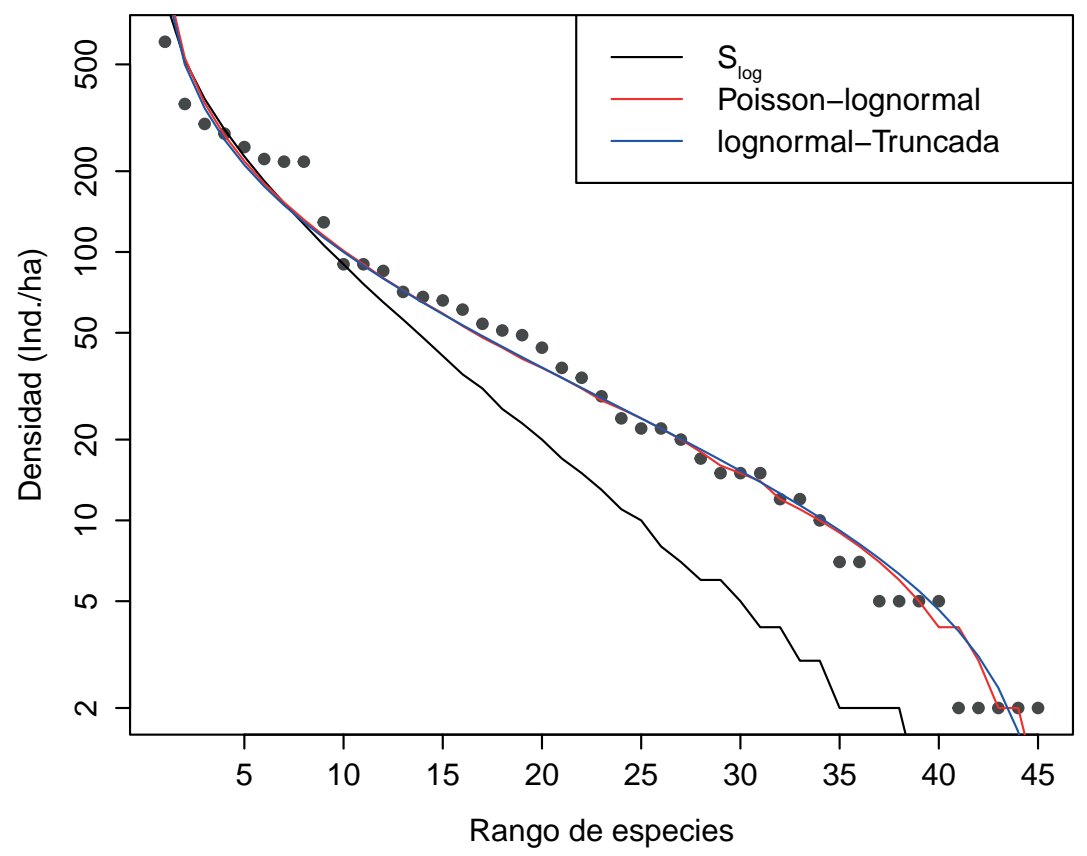

Fig. 2. Curva observada de rango/abundancia de las especies para el matorral submontano contiguo al área metropolitana de Monterrey, México, y funciones ajustadas a esta curva.

\section{DISCUSIÓN}

Riqueza y estructura

La riqueza registrada en el matorral submontano contiguo a la porción occidental del área metropolitana de Monterrey (45 especies) es similar a las registradas por Canizales-Velázquez et al. (2009) y superior a los resultados obtenidos por García y Jurado (2008), quienes también evaluaron una comunidad del matorral submontano en otras áreas de Nuevo León. La familia Fabaceae y el género Acacia fueron los más representativos, coincidiendo con lo reportado por García y Jurado (2008), Canizales-Velázquez et al. (2009) y Estrada-Castillón et al. (2012), quienes evaluaron comunidades maduras de matorral submontano. El alto número de taxa registrados probablemente pueda ser atribuido a que la estructura de los matorrales en general es compleja (MacGillivray y Grime, 1995; Humphries et al., 1995).

Los valores de los índices de Margalef $\left(D_{\mathrm{Mg}}=6.02\right)$ y Shannon $\left(H^{\prime}=3.02\right)$ indican que se trata de una comunidad vegetal diversa, comparada con otros ma- 
torrales que se desarrollan en el noreste de México (Jiménez et al., 2013; Mora et al., 2013). Mora et al. (2013) estudiaron una comunidad madura del matorral espinoso tamaulipeco (MET), registrando valores del índice de Margalef de 2.26 y de Shannon de 1.94. González-Rodríguez et al. (2010) y Ramírez-Lozano et al. (2013) también evaluaron comunidades maduras del MET, ambos obteniendo guarismos del índice de Shannon de 2.40. Mata et al. (2014) evaluaron comunidades maduras de tres matorrales del noreste de México, un desértico micrófilo, un desértico rosetófilo y un submontano, y registraron cifras del índice de Margalef de 2.59, 2.52 y 2.50 respectivamente, y del índice de Shannon de 1.90, 1.89 y 1.88, respectivamente.

Un factor importante de por qué la comunidad evaluada presenta una diversidad alta puede ser el que la zona de estudio se ubica cercana a un área natural protegida, donde las actividades antropogénicas son mínimas, factor que influye en esta riqueza vegetal. De hecho, se ha demostrado que en áreas con disturbios existe una reducción en la diversidad de especies (e.g., Sánchez-Velásquez et al., 2002; Sagar y Singh, 2005; Verzino et al., 2005).

La comunidad evaluada presenta elementos florísticos de las planicies del sur de Texas o de la planicie del Río Bravo (Briones y Villarreal, 2001). Sin embargo, de acuerdo con los parámetros estructurales de las especies, Acacia amentacea y Zanthoxylum fagara fueron las que presentaron el mayor valor de importancia, siendo éstas dos las plantas más abundantes registradas en otros trabajos sobre matorral submontano del centro de Nuevo León (García y Jurado, 2008; Canizales-Velázquez et al., 2009; Estrada-Castillón et al., 2012). Acacia amentacea y Zanthoxylum fagara, elementos estructuralmente importantes en el matorral submontano, también lo son en la comunidad contigua de matorral espinoso tamaulipeco (Estrada et al. 2005; Domínguez et al., 2013). Cabe mencionar que las comunidades vegetales del matorral submontano y matorral espinoso tamaulipeco mencionadas corresponden a biocenosis maduras, características de estados sucesionales avanzados.

Curvas de rango/abundancia de especies

Las especies de la comunidad vegetal evaluada mostraron relaciones de sus rangos y abundancias de tipo log normal. Este tipo de distribución es el más común en comunidades vegetales sin disturbio (García, 1995; Zacarías-Eslava et al., 2011; Long et al., 2012; Martella et al., 2012; Matthews y Whittaker, 2014). Existe una sola especie muy abundante, la cual destaca por su abundancia alta (Acacia amentacea), mientras que otras cinco (Diospyros palmeri, Prosopis laevigata, Casimiroa greggii, Echinocereus pentalophus y Nicotiana glauca) son raras. Magurran (2004) menciona que tal 
modelo es indicativo de comunidades complejas y debido a que muchos ensambles naturales presentan típicamente semejante tipo de curvas, cualquier desviación de este patrón es considerado indicativo de perturbación.

\section{CONCLUSIONES}

De acuerdo con los resultados obtenidos en la presente investigación, se destacan las siguientes conclusiones: (1) la comunidad estudiada presenta una alta riqueza y una alta diversidad de especies en comparación con otras asociaciones vegetales de clima árido y semiárido del noreste de México, (2) la curva de rango/abundancia de las especies se ajustó bien a una función log-normal, distribución característica en la mayoría de las comunidades vegetales maduras y en buen estado de conservación, y (3) las familias con mayor importancia por su contribución a la comunidad son Fabaceae, Rutaceae, Asteraceae y Verbenaceae, mientras que las especies más importantes son Acacia amentacea y Zanthoxylum fagara. La investigación generó información cuantitativa del matorral submontano contiguo al área metropolitana de Monterrey, México, la cual se encuentra en una fase de sucesión ecológica madura. Debido a que esta comunidad vegetal se encuentra muy cercana a una gran zona urbana, es importante proponer estrategias para su conservación, de tal manera que los servicios ambientales que proporciona (hábitat de la biodiversidad, cubierta vegetal, captura de carbono, belleza paisajística, etc.) puedan seguirse aprovechando en el futuro.

\section{AGRADECIMIENTOS}

Los autores agradecen el apoyo de la Ing. María Gabriela Bazaldua Rodríguez en las actividades del campo, a la Ing. Elizabeth Mata Balderas por el apoyo logístico y a los dos árbitros anónimos por sus acertadas recomendaciones y sugerencias que mejoraron la calidad del escrito.

\section{LITERATURA CITADA}

Anónimo. 2002. Resumen del programa de manejo del área natural protegida en la categoría de zona sujeta a conservación ecológica denominada “Sierra Las Mitras”. Periódico Oficial. Monterrey, Nuevo León, México. Miércoles 27 de marzo de 2002. pp. 29-39. 
Alanís-Rodríguez et al.: Estructura y diversidad del matorral submontano de Monterrey, México

Briones, O. L. y J. Á. Villarreal. 2001. Vegetación y flora de un ecotono entre las provincias del altiplano y de la planicie costera de noreste de México. Acta Bot. Mex. 55: 3967.

Briones, O. L. 1991. Sobre la flora, vegetación y fitogeografía de la Sierra de San Carlos, Tamaulipas. Acta Bot. Mex. 16: 15-43.

Canizales-Velázquez, P. A., E. Alanís-Rodríguez, R. Aranda-Ramos, J. M. Mata-Balderas, J. Jiménez-Pérez, G. Alanís-Flores, J. I. Uvalle-Sauceda y M. G. Ruíz-Bautista. 2009. Caracterización estructural del matorral submontano de la Sierra Madre Oriental, Nuevo León. Rev. Chapingo Ser. Cie. 15(2): 115-120.

Domínguez, T. G., H. González, R. G. Ramírez, A. E. Estrada, I. Cantú, M. V. Gómez, J. Á. Villarreal, M. Socorro y G. Alanís. 2013. Diversidad estructural del matorral espinoso tamaulipeco durante las épocas seca y húmeda. Rev. Mex. Cien. For. 4(17): 106-123.

Estrada, E., J. Á. Villarreal y E. Jurado. 2005. Leguminosas del norte del estado de Nuevo León, México. Acta Bot. Mex. 73: 1-18.

Estrada-Castillón, E., J. Á. Villarreal-Quintanilla, E. Jurado-Ybarra, C. Cantú-Ayala, M. A. García-Aranda, J. Sánchez-Salas, J. Jiménez-Pérez y M. Pando-Moreno. 2012. Clasificación, estructura y diversidad del matorral submontano adyacente a la planicie costera del Golfo Norte en el noreste de México. Bot. Sci. 90(1): 37-52.

Farina, A. 1998. Principles and methods in landscape ecology. Towards a science of the landscape. Ed. Chapman and Hall. Londres, UK. 235 pp.

Fernández N., R. y J. A. Colmenero. 1997. Notas sobre la vegetación y flora de San Joaquín, Querétaro, México. Polibotánica 4: 10-36.

Forman R., T. T. y M. Godron M. 1986. Landscape ecology. John Wiley and Sons. Nueva York, USA. 619 pp.

Forman, R. T. 1995. Land mosaics: the ecology of landscapes and regions. Cambridge University Press. Cambridge, UK. 656 pp.

García, E. 1988. Modificaciones al régimen de clasificación climática de Köppen, México. Instituto de Geografía, Universidad Nacional Autónoma de México. México, D.F., México. 90 pp.

García, J. y E. Jurado. 2008. Caracterización del matorral con condiciones prístinas en Linares N.L., México. Ra Ximhai 4(1): 1-21.

García, R. 1995. Diversidad florística de los petenes de Campeche. Acta Bot. Mex. 31: 73-84.

Gómez, S. G., A. Zárate L., J. Valdés y J. Á. Villarreal. 2011. Cambio de uso de suelo del matorral submontano de rosáceas y áreas adyacentes de la Sierra de Zapalinamé, Saltillo, Coah., México. Revista Agraria, Nueva Época 8(1): 24-29.

González-Costilla O., J. Jiménez-de Azcárate, J. García-Pérez y J. R. Aguirre-Rivera. 2007. Flora vascular de la Sierra de Catorce y territorios adyacentes, San Luis Potosí, México. Acta Bot. Mex. 78: 1-38.

González-Rodríguez, H., R. G. Ramírez-Lozano, I. Cantú-Silva, M. V. Gómez-Meza y J. I. Uvalle-Sauceda. 2010. Composición y estructura de la vegetación en tres sitios del estado de Nuevo León, México. Polibotánica 29: 91-106.

Humphries, C.J., P. H. Williams y R. I. Vane-Wright. 1995. Measuring biodiversity value for conservation. Annu. Rev. Ecol. Syst. 26: 93-111. 
Jiménez, J., E. Alanís, M. A. González, O.A. Aguirre y E. J. Treviño. 2013. Characterizing regeneration of woody species in areas with different land-history tenure in the Tamaulipan thornscrub, Mexico. Southwest. Nat. 58(3): 299-304.

Long, W., X. Yang y D. Li. 2012. Patterns of species diversity and soil nutrients along a chronosequence of vegetation recovery in Hainan Island, South China. Ecol. Res. 27(3): 561-568.

MacGillivray C. W. y J. P. Grime. 1995. Testing predictions of the resistance and resilience of vegetation subjected to extreme events. Funct. Ecol. 9: 640-649.

Magurran, A. E. 2004. Measuring biological diversity. Blackwell, Cambridge, Massachusetts, USA. $256 \mathrm{pp}$.

Margalef, R. 1958. Information theory in ecology. Gen. Syst. 3: 36-71.

Martella, M., E. Trumper, L. M. Bellis, D. Renison, P. Giordano, G. Bazzano y R. Gleisser. 2012. Manual de ecología. Evaluación de la biodiversidad. Reduca (Biología) Ser. Ecol. 5(1): 71-115.

Mata, M., E. Treviño, A. Valdecantos, J. Jiménez, O. Aguirre, E. Alanís y R. Foroughbackhch. 2014. Diversidad y composición vegetal de matorrales en el Valle de Santa Catarina, en el Noreste de México. Rev. Iberoam. Cien. 1(3): 3-15.

Matthews, T. J. y R. J. Whittaker. 2014. Fitting and comparing competing models of the species abundance distribution: assessment and prospect. Front. Biogeogr. 6(2):67-82.

Mora, C. A., E. Alanís, J. Jiménez, M.A. González, J. I. Yerena y L. G. Cuellar. 2013. Estructura, composición florística y diversidad del matorral espinoso tamaulipeco, México. Ecol. Apl. 12(1): 29-34.

Moreno, C. E. 2001. Métodos para medir la biodiversidad. M\&T-Manuales y Tesis SEA. Vol. 1. Zaragoza, España. 84 pp. (http://tuxchi.iztacala.unam.mx/disweb/demo_ecologia/ pdfs/libros/mantes1.pdf).

Mostacedo, B. y T. S. Fredericksen. 2000. Manual de métodos básicos de muestreo y análisis en ecología vegetal. BOLFOR. Santa Cruz, Bolivia. 87 pp.

Mueller-Dombois, D. y H. Ellenberg. 1974. Aims and methods of vegetation ecology. John Wiley and Sons, Nueva York, USA. 54 pp.

Muller, C. H. 1939. Relations of the vegetation and climatic types in Nuevo Leon, Mexico. Am. Midl. Nat. 21: 687-729.

Naveh, Z. y A. S. Lieberman. 1984. Landscape ecology. Theory and application. SpringerVerlag, Nueva York, USA. 360 pp.

Palacio, J. L., G. Bocco, A. Velázquez, J. F. Mas, F. Takaki-Takaki, A. Victoria, L. LunaGonzález, G. Gómez-Rodríguez, J. López-García, M. Palma-Muñoz, I. TrejoVázquez, A. Peralta-Higuera, J. Prado-Molina, A. Rodríguez-Aguilar, R. MayorgaSaucedo y F. González-Medrano. 2000. La condición actual de los recursos forestales en México: resultados del inventario forestal nacional 2000. Bol. Inst. Geogr. 43: 183-203.

Potts, J. M. y J. Elith. 2006. Comparing species abundance models. Ecol. Modell. 199: 153163.

Prado, P. I. y M. D. de Miranda. 2014. Fitting species abundance models with maximum likelihood. Theoretical Ecology Lab. Revisado en diciembre de 2014. http://diva. ms.unimelb.edu.au/web/packages/sads/vignettes/sads_intro.pdf 
R Development Core Team. 2011. R: A language and environment for statistical computing. R Foundation for Statistical Computing, Viena, Austria. http://www.R-project.org/.

Ramírez-Lozano R., T. G. Domínguez-Gómez, H. González-Rodríguez, I. Cantú-Silva, M. V. Gómez-Meza, J. I. Sarquís-Ramírez y E. Jurado. 2013. Composición y diversidad de la vegetación en cuatro sitios del noreste de México. Madera y Bosques 19: 59-72.

Rojas-Mendoza, P. 1965. Generalidades sobre la vegetación del estado de Nuevo León y datos acerca de su flora. Tesis de doctorado. Facultad de Ciencias, Universidad Nacional Autónoma de México, México, D.F., México. 124 pp.

Rzedowski, J. 1978. Vegetación de México. Limusa, México, D.F., México. 432 pp.

Rzedowski, J. 1992. Diversidad y origen de la flora fanerógama de México. Ciencias 6: 4756.

Sagar, R. y J. S. Singh. 2005. Structure, diversity, and regeneration of tropical dry deciduous forest of northern India. Biodivers. Conserv. 14(4): 935-959.

Salinas-Rodríguez, M., E. Estrada y J. Á. Villarreal-Quintanilla. 2013. Flora and phytogeography of the Cañón de Iturbide, Nuevo León, México. J. Bot. Res. Inst. Tex. 7(2): 803-819.

Sánchez-Velásquez, L. R., G. Hernández-Vargas, M. A. Carranza, M. R. Pineda-López, R. Cuevas y F. Aragón. 2002. Estructura arbórea del bosque tropical caducifolio usado para la ganadería extensiva en el norte de la Sierra de Manantlán, México. Antagonismo de usos. Polibotánica 13: 25-46.

Spellerberg, l. F y J. W. D. Sawyer. 1999. An introduction to applied biogeography. Cambridge University Press, Cambridge, UK. 260 pp.

Verzino, G, J. Joseau, M. Dorado, E. Gellert, S Rodríguez Reartes y R. Nóbile. 2005. Impacto de los incendios sobre la diversidad vegetal, sierras de Córdoba, Argentina. Ecol. Apl. (4)1-2: 25-34.

Whittaker, R. H. 1972. Evolution and measurement of species diversity. Taxon 21: 213-251. Yablokov, A. V. y S. A. Ostroumov. 1989. Conservación de la naturaleza viva. Vneshtorgizdat, San Petersburgo, URSS. 237 pp.

Zacarías-Eslava, L. E., G. Cornejo-Tenorio, J. Cortés-Flores, N. González-Castañeda y G. Ibarra-Manríquez. 2011. Composición, estructura y diversidad del cerro El Águila, Michoacán, México. Rev. Mex. Bodivers. 82(3): 854-869. 


\section{APÉNDICE}

Nombre científico, nombre común, forma biológica, orden y familia de las especies registradas en el área de estudio (ordenadas alfabéticamente por nombre científico).

\begin{tabular}{|c|c|c|c|}
\hline Especie & Nombre común & Forma biológica & Familia \\
\hline Acacia amentacea DC. & chaparro prieto & arbusto & Fabaceae \\
\hline Acacia berlandieri Benth. & guajillo & árbol & Fabaceae \\
\hline Acacia farnesiana (L.) Willd. & huizache & arbusto & Fabaceae \\
\hline Acacia greggii A. Gray & uña de gato & arbusto & Fabaceae \\
\hline Agave lecheguilla Torr. & lechuguilla & suculenta & Agavaceae \\
\hline $\begin{array}{l}\text { Bernardia myricifolia (Scheele) } \\
\text { Benth. \& Hook. f. }\end{array}$ & oreja de ratón & arbusto & Euphorbiaceae \\
\hline Caesalpinia mexicana A. Gray & árbol del potro & arbusto & Fabaceae \\
\hline $\begin{array}{l}\text { Casimiroa greggii (S. Watson) F. } \\
\text { Chiang }\end{array}$ & chapote amarillo & árbol & Rutaceae \\
\hline $\begin{array}{l}\text { Castela erecta subsp. texana (Torr. } \\
\text { \& A. Gray) Cronquist }\end{array}$ & chaparro amargo & arbusto & Simaroubaceae \\
\hline Celtis laevigata Willd. & palo blanco & árbol & Ulmaceae \\
\hline Celtis pallida Torr. & granjeno & arbusto & Ulmaceae \\
\hline $\begin{array}{l}\text { Citharexylum berlandieri B.L. } \\
\text { Rob. }\end{array}$ & revientacabras & arbusto & Verbenaceae \\
\hline Condalia hookeri M.C. Johnst. & brasil & árbol & Rhamnaceae \\
\hline Cordia boissieri A. DC. & anacahuita & árbol & Boraginaceae \\
\hline Croton ciliatoglandulifer Ortega & falsa salvia & arbusto & Euphorbiaceae \\
\hline Diospyros palmeri Eastw. & chapote prieto & árbol & Ebenaceae \\
\hline Diospyros texana Scheele & chapote manzano & árbol & Ebenaceae \\
\hline $\begin{array}{l}\text { Ebenopsis ebano (Berland.) } \\
\text { Barneby \& J.W. Grimes }\end{array}$ & ébano & árbol & Fabaceae \\
\hline $\begin{array}{l}\text { Echinocereus enneacanthus } \\
\text { Engelm. }\end{array}$ & pitayo mayor & suculenta & Cactaceae \\
\hline $\begin{array}{l}\text { Echinocereus pentalophus (DC.) } \\
\text { Haage }\end{array}$ & pitayo pequeño & suculenta & Cactaceae \\
\hline $\begin{array}{l}\text { Ehretia anacua (Terán \& Berland.) } \\
\text { I.M. Johnst. }\end{array}$ & anacua & arbusto & Boraginaceae \\
\hline Eysenhardtia texana Scheele & vara dulce & arbusto & Fabaceae \\
\hline $\begin{array}{l}\text { Ferocactus haematacanthus Bravo } \\
\text { ex Backeb. \& F.M. Knuth }\end{array}$ & $\begin{array}{l}\text { biznaga de } \\
\text { anzuelitos }\end{array}$ & suculenta & Cactaceae \\
\hline
\end{tabular}


Apéndice. Continuación

\begin{tabular}{|c|c|c|c|}
\hline Especie & Nombre común & Forma biológica & Familia \\
\hline Forestiera angustifolia Torr. & panalero & arbusto & Oleaceae \\
\hline Fouquieria splendens Engelm. & ocotillo & arbusto & Fouquieriaceae \\
\hline Fraxinus greggii A. Gray & fresno silvestre & árbol & Oleaceae \\
\hline Guaiacum angustifolium Engelm. & guayacán & arbusto & Zygophyllaceae \\
\hline $\begin{array}{l}\text { Gymnosperma glutinosum } \\
\text { (Spreng.) Less. }\end{array}$ & escobilla & arbusto & Asteraceae \\
\hline $\begin{array}{l}\text { Havardia pallens (Benth.) Britton } \\
\text { \& Rose }\end{array}$ & tenaza & arbusto & Fabaceae \\
\hline $\begin{array}{l}\text { Helietta parvifolia (A. Gray) } \\
\text { Benth. }\end{array}$ & barreta & arbusto & Rutaceae \\
\hline Jatropha dioica Cerv. & sangre de drago & arbusto & Euphorbiaceae \\
\hline $\begin{array}{l}\text { Karwinskia humboldtiana } \\
\text { (Schult.) Zucc. }\end{array}$ & coyotillo & arbusto & Rhamnaceae \\
\hline Lantana macropoda Torr. & lantana & arbusto & Verbenaceae \\
\hline $\begin{array}{l}\text { Leucophyllum frutescens } \\
\text { (Berland.) I.M. Johnst. }\end{array}$ & cenizo & arbusto & Scrophulariaceae \\
\hline Lippia graveolens Kunth & orégano & arbusto & Verbenaceae \\
\hline $\begin{array}{l}\text { Mammillaria prolifera (Mill.) } \\
\text { Haw. }\end{array}$ & biznaguita & suculenta & Cactaceae \\
\hline Mimosa malacophylla A. Gray & charrasquillo & arbusto & Fabaceae \\
\hline Nicotiana glauca Graham & tabaquillo & arbusto & Solanaceae \\
\hline $\begin{array}{l}\text { Opuntia engelmannii (Salm-Dyck) } \\
\text { ex Engelm. }\end{array}$ & nopal cuijo & suculenta & Cactaceae \\
\hline $\begin{array}{l}\text { Prosopis laevigata (Humb. \& } \\
\text { Bonpl. ex Willd.) M.C. Johnst. }\end{array}$ & mezquite & árbol & Fabaceae \\
\hline $\begin{array}{l}\text { Senna bauhinioides (A. Gray) H.S. } \\
\text { Irwin \& Barneby }\end{array}$ & senna & arbusto & Fabaceae \\
\hline $\begin{array}{l}\text { Sophora secundiflora (Ortega) } \\
\text { Lag. ex DC. }\end{array}$ & colorín & arbusto & Fabaceae \\
\hline Yucca filifera Chabaud & palma china & arbusto & Agavaceae \\
\hline Zanthoxylum fagara (L.) Sarg. & colima & arbusto & Rutaceae \\
\hline $\begin{array}{l}\text { Ziziphus obtusifolia (Hook. ex } \\
\text { Torr. \& A. Gray) A. Gray }\end{array}$ & clepe & arbusto & Rhamnaceae \\
\hline
\end{tabular}

